\title{
A GENERALIZATION OF INTEGRAL INVARIANTS
}

\section{E. VIDAL ABASCAL}

The integral invariants in the sense of Poincaré-Cartan refer to integral expressions in connection with the integral curves of a system of ordinary differential equations of first order in $n$ variables. The object of this note is to generalize the notion of integral invariant to the integral varieties of a completely integrable system of Pfaff, as well as to extend to these varieties the integral relation of invariance given for the system of curves by Lichnerowicz [3], ${ }^{1}$ and other theorems including those relating to curves as particular cases. Some applications are given.

\section{INTEGRAL INVARIANTS-INTEGRAL RELATIONS OF INVARIANCE}

1. Definition of integral invariants. Given a completely integrable system of Pfaff

$$
\omega_{1}=0, \quad \omega_{2}=0, \quad \cdots, \quad \omega_{h}=0
$$

in $m$ parameters $(h<m)$, let us consider the ensemble of integral varieties $V_{m-h}$ of the system (1.I) of $m-h$ dimension, that fill the representative space $R$ of $m$ dimensions.

Let us consider an arbitrary domain $D_{0}^{(p)}$ of $p \leqq h$ dimensions and let us suppose that in some way or another we displace the different points of $D_{0}^{(p)}$ on the integral varieties of the system (1.I) till we obtain another domain of $p$ dimensions $D_{1}^{(p)}$.

We shall call $T^{(p+1)}$ the domain of $(p+1)$ dimensions generated by arcs of integral curves, each being limited by the points of $D_{0}^{(p)}$ and $D_{1}^{(p)}$; and we shall call $N^{(p)}$ the domain generated by the boundary of $D_{0}^{(p)}$ when displaced to $D_{1}^{(p)}$.

Let $\Omega$ be an exterior differential form of degree $p$ belonging in $R$ to the class $C^{(2)}$.

DEFINITION. We shall say that $\int \Omega$ is an absolute integral invariant for the system (1.I), if whatever may be the domain $D_{0}^{(p)}$ and the domain $D_{1}^{(p)}$ which is deduced

$$
\int_{D_{0}^{(p)}} \Omega=\int_{D 1}{ }^{(p)} \Omega .
$$

If the relation (2.I) holds only if $D_{0}^{(p)}$ (and therefore $D_{1}^{(p)}$ ) is closed, $\Omega$ will be said to be a relative integral invariant.

Received by the editors November 14, 1958.

1 Numbers enclosed by brackets refer to the Bibliography at the end of the paper. 
2. Definition of integral relations of invariance. Let $\Omega$ be an exterior differential form of degree $(p+1)$ belonging in $R$ to class $C^{(2)}$.

Definition. We shall say that $\Omega$ yields for system (1.I) an integral relation of absolute invariance if whatever may be the domain $D_{0}^{(p)}$ and the domain $T^{(p+1)}$ which is deduced

$$
\int_{T^{(p+1)}} \Omega=0
$$

$\Omega$ yields an integral relation of relative invariance if for the relation (3.I) to hold, the domain $D_{0}^{(p)}$ must be closed.

\section{Integral relation of absolute invariance.}

Theorem I. For $\Omega$ to give rise to an integral relation of absolute invariance of the differential system (1.I) it is necessary and sufficient that only differential forms of first integrals of that system intervene in the form $\Omega$ as differential forms.

Proof. Let

$$
\phi_{i}\left(a_{1}, a_{2}, \cdots, a_{m}\right)=\xi_{i} \quad(i=1,2, \cdots, h)
$$

be the first $h$ independent integrals of (1.I); this system is locally equivalent to

$$
d \xi_{1}=0, d \xi_{2}=0, \cdots, d \xi_{h}=0 .
$$

If $\Omega$ defines an integral relation of absolute invariance with respect to the integral varieties $V_{m-h}$ of the Pfaffian system (1.I), then we say that $\Omega$ contains only differentials of first integrals of (1.I). The condition is necessary: For if $\Omega$ defines an integral relation of invariance with respect to the varieties $V_{m-h}$, that means, by definition, it defines that relation with respect to any system of curves contained in those varieties (that is to say: a curve on condition that if it has a point on a variety, the whole of it is contained in that variety; and to form a system means that one of those curves passes through each point of the space considered).

But $m-h$ systems of curves on the varieties can be written in the form (vid. Cartan [1, p. 40])

$$
\text { (5.I) } \begin{array}{r}
\frac{d \xi_{1}}{0}=\frac{d \xi_{2}}{0}=\cdots=\frac{d \xi_{h}}{0}=\frac{d y_{h+1}}{0}=\cdots= \\
=\frac{d y_{s}}{Y_{s}}=\cdots=\frac{d y_{m}}{0}, \\
(s=h+1, \cdots, m) .
\end{array}
$$

By the theorem demonstrated by Lichnerowicz [3], the form that gives rise to an integral relation of absolute invariance relative to this 
system will only contain first integrals as differentials, that is to say that following $m-1$ differentials will enter in the form:

$$
d \xi_{1}, d \xi_{2}, \cdots, d \xi_{h}, d y_{h+1}, \cdots, d y_{s-1}, d y_{s+1}, \cdots, d y_{m} .
$$

But as the form must define the relation of invariance with respect to all these systems $(s=h+1, h+2, \cdots, m)$, only those common to all of them will be able to enter as differentials, that is to say

$$
d \xi_{1}, d \xi_{2}, \cdots, d \xi_{h}
$$

as we wanted to demonstrate.

$\Omega$ will be written, therefore, in the form

$$
\Omega=\sum A_{i_{1} i_{2} \cdots i_{p+1}} d \xi_{i_{1}} \wedge d \xi_{i_{2}} \wedge \cdots \wedge d \xi_{i_{p+1}} .
$$

Let us see now that the condition is sufficient. In fact, if only differential of first integrals enter the form, that is to say its expression is (6.I), we want to prove that it satisfies the relation

$$
\int_{T^{(p+1)}} \Omega=0
$$

To this purpose, let us represent parametrically the domain $T^{(p+1)}$ by means of the parameters $\left(v, \alpha_{1}, \cdots, \alpha_{p}\right)$. In accordance with the definition given, we suppose the domain $T^{(p+1)}$ generated by a domain $D_{0}^{(p)}$ that moved, each point sliding on the variety $V_{m-h}$ where it finds itself, to the domain $D_{1}^{(p)} \cdot\left(\alpha_{1}, \cdots, \alpha_{p}\right)$ determine the point of the domain $D_{0}^{(p)}$, the variable $v$ determines the point in its movement on $V_{m-h}$.

On passing to the parameter representation of the expression (6.I) referring to these variables, to each expression

$$
d \xi_{i_{1}} \wedge d \xi_{i_{2}} \wedge \cdots \wedge d \xi_{i_{p+2}}
$$

corresponds a determinant the elements of which in the first row

$$
\frac{d \xi_{i_{1}}}{d v}, \frac{d \xi_{i_{2}}}{d v}, \ldots, \frac{d \xi_{i_{p+1}}}{d v}
$$

are all null, since each ensemble of values $\xi_{1}, \cdots, \xi_{h}$ determines a variety and $v$ varies within each variety, the values of $\xi_{i}$ do not depend on $v$.

4. Integral relations of invariance and integral invariants. We deduce relations similar to those given for curves, between the notions of integral relation of invariance and integral invariant. 
THEOREM II. If $d \Omega$ yields an integral relation of relative invariance, the form $\Omega$ defines a relative integral invariant; if, besides, $\Omega$ also yields an absolute integral relation of invariance, then $\Omega$ defines an absolute integral invariant.

Proof. To prove the first part, if we suppose $D_{0}^{(p)}$ and $D_{1}^{(p)}$ closed limiting the domain $T^{(p+1)}$, by Stokes' theorem, we deduce

$$
\int_{D_{0}^{(p)}} \Omega-\int_{D_{1}^{(p)}} \Omega=\int_{T^{(p+1)}} d \Omega=0
$$

which proves that $\int \Omega$ is a relative integral invariant.

If $\Omega$ yields an absolute integral relation of invariance by Theorem I, the system associated with $\Omega$ is satisfied if we take into account the equations (1.I). If besides $d \Omega$ also yields a relative integral relation of invariance for the system (1.I), then the characteristic system of $\Omega$, that is formed by the union of the associated system of $\Omega$ and $d \Omega$ is satisfied by equations (1.I), and $\Omega$ defines an absolute integral invariant with respect to this last system, as we wanted to prove.

Conversely, if $\int \Omega$ is a generalized absolute invariant, it is an ordinary absolute invariant in the sense of Cartan with respect to any system of curves drawn on the integral varieties of (1.I); therefore $\Omega$ has

$$
\omega_{1}=0, \omega_{2}=0, \cdots, \omega_{h}=0
$$

as characteristic system, and $V_{m-h}$ are the characteristic varieties of the form $\Omega$.

Given the domain $D_{0}^{(p)}$ if a point describes the corresponding trajectory $C$ on the variety $V_{m-h}$, there exists a system of curves on the integral varieties in which $C$ participates and for which $\int \Omega$ is an invariant. To give this curve will be to give the initial conditions.

THEOREM III. If $\int \Omega$ is an absolute integral invariant of degree $h$ with respect to a set of integral varieties $V_{m-h}$ (of $m-h$ dimensions) of the system (1.I), then

$$
d \Omega=0 .
$$

Proof. If $\Omega$ defines an integral invariant with respect to the varieties $V_{m-h}$ of $m-h$ dimensions, solutions of the (1.I), $\Omega$ is invariant with respect to any system of curves drawn on those surfaces corresponding to a wholly integrable system. Let us consider $m-h$ systems of curves on the varieties independent from each other; the variation $\delta \Omega$ is null in these $m-h$ directions and in the $h$ directions corresponding to the differentials entering in the form $\Omega$, which proves that the 
variation of $\Omega$ is null in $m$ independent directions. This theorem generalizes the one established by Cartan [1] for absolute invariants of $m-1$ degree with respect to a system of curves.

THEOREM IV. If we have the completely integrable system of Pfaff (1.I), the differential form of $h$ degree

$$
\Omega=\omega_{1} \wedge \omega_{2} \wedge \cdots \wedge \omega_{h}
$$

if it fulfills the condition

$$
d \Omega=0
$$

is an absolute integral invariant with respect to the integral varieties of system (1.I).

Proof. By Theorem I, it will be sufficient to prove that both $\Omega$ and $d \Omega$ yield integral relations of invariance. The differential form $\Omega$ yields an integral relation of invariance, that is to say $\int_{N}^{(h)} \Omega=0$, $N^{(h)}$ being yielded by the boundary of $D_{0}^{(h)}$ when displaced till $D_{1}^{(h)}$, but the intersection $N^{(h)} \cap V_{m-h}$ are points that are displaced on the varieties and on these varieties $\omega_{1}=\omega_{2}=\cdots=\omega_{h}=0$.

By the hypothesis (8.I), $d \Omega$ also yields an integral relation of invariance. Observe that $\Omega$ being of degree $h$ and the varieties of degree $m-h$, the condition $\int_{T^{(h+1)}} d \Omega=0$ is equivalent to (8.I), as we saw in Theorem III.

\section{Applications}

5. Integral invariants of $m$ degree with respect to a system of curves. Given the system

$$
\frac{d x_{1}}{X_{1}}=\frac{d x_{2}}{X_{2}}=\cdots=\frac{d x_{m}}{X_{m}}=d v
$$

the expression

$$
\Omega=\left(d x_{1}-X_{1} d v\right) \wedge\left(d x_{2}-X_{2} d v\right) \wedge \cdots \wedge\left(d x_{m}-X_{m} d v\right)
$$

will be, from Theorem IV, an absolute integral invariant of degree $m$ in the space of dimension $m+1\left(x_{1}, x_{2}, \cdots, x_{m}, v\right)$, if it fulfills the condition $d \Omega=0$.

EXAMPLE. In a continuous medium in motion, the trajectory of each molecule can be considered as the solution of a system of differential equations

$$
\frac{d x}{d t}=u, \quad \frac{d y}{d t}=v, \quad \frac{d z}{d t}=w,
$$


therefore

$$
\int \Omega=\int(d x-u d t) \wedge(d y-v d t) \wedge(d z-w d t)
$$

will be an absolute integral invariant which represents the measure of the whole of trajectories. If each molecule is supposed of density $\rho(x, y, z)$, we deduce, the mass expressed in its kinematic aspect

$$
\iiint \rho(d x d y d z-u d y d z d t-v d z d x d t-w d x d y d t) .
$$

From $d \Omega=0$, we deduce the condition

$$
\frac{\partial \rho}{\partial t}+\frac{\partial(\rho u)}{\partial t}+\frac{\partial(\rho v)}{\partial t}+\frac{\partial(\rho w)}{\partial t}=0
$$

called conservation of matter (vid. Cartan [1]).

6. Relation between integral invariants and integral geometry. The generalizations established and the theorems demonstrated let us extend integral geometry to the measure of ensembles of integral varieties of a completely integrable Pfaffian system.

Given the completely integrable Pfaffian system (1.I), the expression

$$
\int \Omega=\int \omega_{1} \wedge \omega_{2} \wedge \cdots \wedge \omega_{h}
$$

extended over a domain $D_{0}^{(h)}$ having a point on each of the integral varieties $V_{m-h}$ of an ensemble of them, of $h$ dimensions, if $d \Omega=0$ holds, fulfills both conditions given by Chern [2] to be a measure: Invariance under a transformation of co-ordinates and invariance if each point of $D_{0}^{(h)}$ is displaced on the corresponding variety to another domain $D_{1}^{(h)}$. We have proved that this last condition corresponds to $\Omega$ 's being a generalized absolute integral invariant. The varieties being of dimension $m-h$ and $\Omega$ of degree $h$, this means, from Theorem III,

$$
d \Omega=0 .
$$

If we suppose that the forms (1.I) are relative components of a group, and that in accordance with the equations of structure of Maurer-Cartan

$$
d \omega_{i}=\sum_{l<k} C_{l k}^{i} \omega_{l} \wedge \omega_{k}, \quad C_{l k}^{i}+C_{k l}^{i}=0
$$


holds, then. Chern [2] has demonstrated that in order that (3.II) be a measure, it is necessary and sufficient that

$$
\sum_{i=1}^{h} C_{i k}^{i}=0, \quad k=h+1, \cdots, r .
$$

But Santaló [5] has proved that this last condition, in this case, is equivalent to condition (4.II); with which we obtain that the fulfillment of both conditions is the same as to suppose that $\int \Omega$ is a generalized absolute integral invariant, which means they are included in a particular case of Theorem III.

\section{REFERENCES}

1. E. Cartan, Leçons sur les invariants intégraux, Hermann, Paris, 1922.

2. S. S. Chern, On integral geometry in Klein spaces, Ann. of Math. vol. 43 (1942) pp. $178-189$.

3. A. Lichnerowicz, Les relations intégrales d'invariance et leurs applications d la dynamique, Bull. Sci. Math. vol. 70 (1946) pp. 1-14.

4. L. A. Santalo, Integral geometry in projective and affine spaces, Ann. of Math. vol. 51 (1950) pp. 739-755.

5. - Introduction to integral geometry, Actualités Sci. Ind., no. 1198, Hermann, Paris, 1953.

University of Santiago de Compostela 